\title{
$\varepsilon$ - Neighbourhood Median Filters to Remove Speckle Noise from CT - Images
}

\author{
Gnanambal llango \\ Postgraduate and Research Department of \\ Mathematics, \\ Government Arts College (Autonomous), \\ Coimbatore - 641018. Tamilnadu, India.
}

\author{
B. Shanthi Gowri \\ Department of Mathematics, \\ Sri Krishna College of Engineering and \\ Technology, \\ Coimbatore - 641008. Tamilnadu, India.
}

\begin{abstract}
Removal of noise from the medical images is very challenging in image processing. In recent years, technological development has improved significantly in analyzing medical imaging. This paper proposes different filtering techniques for the removal of speckle noise from CT medical images by topological approach. The filters are constructed based on metric topological neighbourhood. The quality of the enhanced images is measured by the statistical quality measures: Root Mean Square Error (RMSE) and Peak Signal to Noise Ratio (PSNR).
\end{abstract}

\section{General Terms}

Metric topology, Filtering technology, Image enhancement.

\section{Keywords}

Metric topological neighbourhood, CT images, speckle noise, RMSE, PSNR.

\section{INTRODUCTION}

Digital image analysis plays a vital role in medical imaging like magnetic resonance imaging, ultra sound imaging, X-ray and computed tomography. Departure of the ideal signal is usually referred to as noise. Noises in such digital images arise during image acquisition and/or transmission. The data dropout noise is generally called as speckle noise. Speckle noise is a multiplicative noise that degrades the visual evaluation in medical imaging. Speckle noise suppression plays a very essential role in diagnosis. The image acquisition devices need despeckling techniques for medical imaging in routine clinical practice. Image filtering is an important technique used for the detection and removal of noise from the digital images. Median filter has been introduced by Turkey in 1970 [1]. It is a non-linear filter used for smoothing the images. Sudha et al [2] recommends a novel thresholding algorithm for denoising speckle noise in ultrasound images with wavelets. An improved adaptive median filtering method for denoising impulse noise was carried out by Mamta Juneja et al [3]. Thangavel et al [4] showed that the M3-filter had performed better than Mean, Median, Max, Min and various other filters. The Hybrid Max filter which performs significantly better than many other existing techniques for removal of speckle noise was shown by Gnanambal Ilango et al [5]. R. Marudhachalam and Gnanambal Ilango [6] proposed different Center Weighted Hybrid filtering techniques for denoising of medical images. The objective of this study is to develop new filtering techniques based on different metric topological neighbourhoods and investigate their performance on CT images. In this experimental work, the different filtering techniques for noise removal were applied on CT image of the liver with speckle noise. The quality of the de-noised image is measured by the statistical quality measures: RMSE and PSNR.

This work is organized as follows: In Section 2, basic definitions are given. Section 3 discusses the various filtering techniques for de-noising the speckle noise in Medical images. Section 4 deals with the proposed new filtering techniques based on Metric topological neighbourhood for de-noising the speckle noise in CT images. Section 5 explains the experimental work done. Section 6 discusses the comparison of results of various new filtering techniques. Section 7 concludes the experimental results.

\section{BASIC DEFINITIONS:}

\subsection{Definition [7]}

A metric on a set $\mathrm{X}$ is a function $\mathrm{d}: \mathrm{X} \times \mathrm{X} \rightarrow \mathrm{R}$ having the following properties:

i. $\quad d(x, y) \geq 0 \quad \forall x, y \in X$; equality holds

ii. $\quad d(x, y)=d(y, x) \quad \forall x, y \in X$.

$$
\text { iff } x=y \text {. }
$$

iii. $\quad d(x, z) \leq d(x, y)+d(y, z) \forall x, y, z \in X$.

Given a metric $\mathrm{d}$ on $\mathrm{X}$, the number $d(x, y)$ is called as the distance between $\mathrm{x}$ and $\mathrm{y}$ in the metric $\mathrm{d}$. For a given $\varepsilon>0$, consider the set

$B_{d}(x, \varepsilon)=\{y / d(x, y)<\varepsilon\}=B(x, \varepsilon)$

of all points $\mathrm{y}$ whose distance from $\mathrm{x}$ is less than $\varepsilon$. Here $B_{d}(x, \varepsilon)$ is called the $\varepsilon$ - ball centered at $\mathrm{x}$. If $\mathrm{d}$ is a metric on the set $\mathrm{X}$, then the collection of all

$\varepsilon$ - balls $B_{d}(x, \varepsilon)$ for $x \in X$ and $\varepsilon>0$, is a basis for a topology on $X$, called metric topology induced by $\mathrm{d}$. A set $\mathrm{U}$ is open in the metric topology induced by $\mathrm{d}$ iff for each $y \in U$, there is a $\delta>0 \ni: B_{d}(y, \delta) \subset U$. If $\mathrm{X}$ is a topological space, $X$ is said to be metrizable if there exists a metric $d$ on the set $X$ that induces the topology of $\mathrm{X}$. A metric space is a metrizable space $X$ together with a specific metric $\mathrm{d}$ that gives the topology of $\mathrm{X}$.

\subsection{Definition [8]}

An image may be defined as a two-dimensional function $\mathrm{f}(\mathrm{x}, \mathrm{y})$, where $\mathrm{x}$ and $\mathrm{y}$ are spatial (plane) coordinates, and the amplitude of $\mathrm{f}$ at any pair of coordinates $(\mathrm{x}, \mathrm{y})$ is called the intensity or gray level of the image at that point. If $x, y$ and the intensity values of $f$ are all finite, discrete quantities, we call the image a digital image. A digital image is composed of a finite number of elements $(x, y)$ each of which 
has a particular location and value. These elements are called picture elements or pixels.

\subsection{Definition}

An image in the positive quadrant is a function $f: W X W \rightarrow[0,1,2, \ldots . N-1]$ in which $N-1$ is a positive whole number belonging to the natural interval $[1,256]$. The functional value of ' $f$ ' at any point $\mathrm{p}(\mathrm{x}, \mathrm{y})$ is called the intensity or gray level of the image at that point and it is denoted by $f(p)$ or $f(x, y)$.

\subsection{Definition [9]}

Let $\mathrm{X}$ be an array of points having positive integer coordinates (x,y), where $1 \leq x \leq M, 1 \leq y \leq N$. The neighbourhood of a point $p \in X$ is a subset of $\mathrm{X}$ which contains an open set containing $\mathrm{p}$. It is denoted by $\mathrm{N}(\mathrm{p})$.

\subsection{Definition}

$$
\begin{aligned}
& \text { Let } S^{2}=\mathrm{S} \mathrm{X} S \\
& =\left\{\begin{array}{c}
(\mathrm{x}, \mathrm{y}) ; \mathrm{x}=0,1,2,3, \ldots \mathrm{M}-1 \\
\text { and } \mathrm{y}=0,1,2,3, \ldots \mathrm{N}-1
\end{array}\right\}
\end{aligned}
$$

be the set of spatial coordinates of a digital image. The discrete topology on $S^{2}$ is the collection of all subsets of $S^{2}$ denoted by $\mathfrak{I}_{S^{2}}$.

\subsection{Definition [9]}

Let $p=\left(x_{1}, y_{1}\right), q=\left(x_{2}, y_{2}\right) \in S^{2}$. Consider the functions

i) $d_{4}: S X S \rightarrow R$, defined by

$d_{4}(p, q)=\left|x_{1}-x_{2}\right|+\left|y_{1}-y_{2}\right|$.

Then $\left(S^{2}, d_{4}\right)$ is a metric space. The metric $d_{4}$ is called City-block metric or Manhattan metric.

ii) $d_{8}: S X S \rightarrow R$, defined by

$$
d_{8}(p, q)=\max \left\{\left|x_{1}-x_{2}\right|,\left|y_{1}-y_{2}\right|\right\} .
$$

Then $\left(S^{2}, d_{8}\right)$ is a metric space. The metric $d_{8}$ is called the Chessboard metric.

\subsection{Definition}

For any metric space $\left(S^{2}, d\right)$, any $p \in S^{2}$, and any $\varepsilon>0$, consider the set $N_{d, \varepsilon}(p)=\{q ; d(p, q)<\varepsilon+1\} . N_{d, \varepsilon}(p)$ is called the (open) $\varepsilon$ - neighbourhood of $p$ in $S^{2}$.

\subsection{Definition}

For any point $\mathrm{p}$, the 4 -neighbourhood of $\mathrm{p}$ denoted by $N_{d_{4}, 1}(p)$ is defined $N_{d_{4}, 1}(p)=\left\{q \in S^{2} ; d_{4}(p, q)<2\right\} . N_{d_{4}, 1}(p)$ is also denoted by $N_{4}(p)$ and the 8 - neighbourhood of $\mathrm{p}$ denoted by $N_{d_{8}, 1}(p)$ is defined as $N_{d_{8}, 1}(p)=\left\{q \in S^{2} ; d_{8}(p, q)<2\right\}$. $N_{d_{8}, 1}(p)$ is also denoted by $N_{8}(p)$.

\subsection{Definition}

For any point $\mathrm{p}$, the 4 - neighbours of $\mathrm{p}$ are $N_{d_{4}, 1}(p)-\{p\}$, and the 8 - neighbours of $\mathrm{p}$ are $N_{d_{8}, 1}(p)-\{p\}$.

\subsection{Definition}

\section{Let $\quad M \subseteq S X S$ where}

$M=\{(x, y \pm k) ;$ where $k=0,1,2,3 \ldots . \quad$ Let $p=\left(x_{1}, y_{1}\right), q=\left(x_{2}, y_{2}\right) \in M$. Consider the function $\quad d_{V}: M \rightarrow R \quad$ defined by $d_{V}(p, q)=\left|x_{1}-x_{2}\right|+\left|y_{1}-y_{2}\right| \cdot\left(M, d_{V}\right)$ is a metric space. For any point $\mathrm{p}$, the $V_{\varepsilon}$ neighbourhood of $\mathrm{p}$ is defined as $N_{d_{v}, \varepsilon}(p)=\left\{q ; d_{v}(p, q)<\varepsilon+1\right\}$. Hence the $V_{1}$ neighbourhood of $\mathrm{p}$ is $N_{d_{v}, 1}(p)=$ $\left\{q ; d_{v}(p, q)<2\right\}$ and the $V_{2}$ neighbourhood of $\mathrm{p}$ is $N_{d_{v}, 2}(p)=\left\{q ; d_{v}(p, q)<3\right\}$. The $V_{1}$ neighbours of $\mathrm{p}$ are $N_{d_{v}, 1}(p)-\{p\}$ and the $V_{2}$ neighbours of $\mathrm{p}$ are $N_{d_{V, 2}}(p)-\{p\}$.

\subsection{Definition}

Let $M \subseteq S X S$ where

$M=\{(x \pm k, y) ;$ where $k=0,1,2,3 \ldots\}$. Let $p=\left(x_{1}, y_{1}\right), q=\left(x_{2}, y_{2}\right) \in M$. Consider the function $d_{H}: M \rightarrow R \quad$ defined by $\quad d_{H}(p, q)=\left|x_{1}-x_{2}\right|+\left|y_{1}-y_{2}\right|$.

$\left(M, d_{H}\right) \quad$ is a metric space. For any point p, the $\varepsilon-H$ neighbourhood of $\mathrm{p}$ is defined as

$N_{d_{H, \varepsilon}}(p)=\left\{q ; d_{H}(p, q)<\varepsilon+1\right\}$. Hence the 1- $H$ neighbourhood of $\mathrm{p}$ is defined as $N_{d_{H, 1}}(p)=$

$\left\{q ; d_{H}(p, q)<2\right\}$ and the $2-H$ neighbourhood of $\mathrm{p}$ is defined as $N_{d_{H, 2}}(p)=\left\{q ; d_{H}(p, q)<3\right\}$. The 1-H neighbours of $\mathrm{p}$ are $N_{d_{H, 1}}(p)-\{p\}$ and the 2- $H$ neighbours of $\mathrm{p}$ are $N_{d_{H, 2}}(p)-\{p\}$.

\subsection{Definition}

Let $M \subseteq S X S$, where

$$
M=\left\{\begin{array}{c}
\{(x+k, y-k)\} \cup\{(x-k, y+k)\} ; \\
\text { where } k=0,1,2,3 \ldots .
\end{array}\right\} .
$$

Let $p=\left(x_{1}, y_{1}\right), q=\left(x_{2}, y_{2}\right) \in M$. Consider the function $\quad d_{L T}: M \rightarrow R \quad$ defined by $d_{L T}(p, q)=\frac{1}{2}\left[\left|x_{1}-x_{2}\right|+\left|y_{1}-y_{2}\right|\right]$.

$\left(M, d_{L T}\right) \quad$ is a metric space. For any point $\mathrm{p}$, the $L T_{\varepsilon}$ neighbourhood of $\mathrm{p}$ is defined as 
$N_{d_{L T}, \varepsilon}(p)=\left\{q ; d_{L T}(p, q)<\varepsilon+1\right\}$. Hence the $L T_{1}$ neighbourhood of $p$ is defined as $N_{d_{L T}, 1}(p)=\left\{q ; d_{L T}(p, q)<2\right\}$ and the $L T_{2}$ neighbourhood of $\mathrm{p}$ is defined as $N_{d_{L T}, 2}(p)=$ $\left\{q ; d_{L T}(p, q)<3\right\} . \quad N_{d_{L T}, 1}(p)$ is also denoted by $L_{3}(p)$. The $L T_{1}$ neighbours of $p$ are $N_{d_{L T}, 1}(p)-\{p\}$ and the $L T_{2}$ neighbours of $\mathrm{p}$ are $N_{d_{L T} 2}(p)-\{p\}$.

\subsection{Definition}

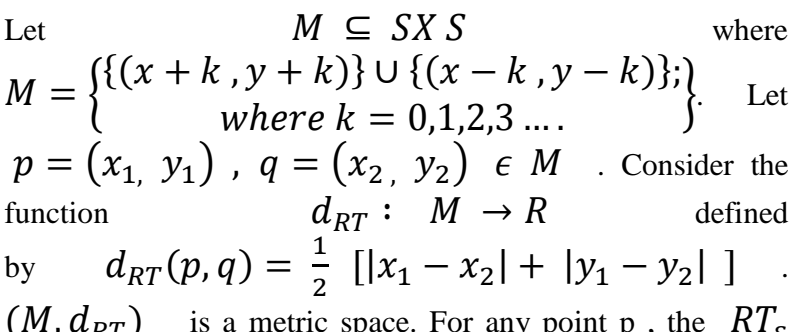
$\left(M, d_{R T}\right)$ is a metric space. For any point $\mathrm{p}$, the $R T_{\varepsilon}$
neighbourhood of $\mathrm{p}$ is defined as $N_{d_{R T} \varepsilon}(p)=$ $\left\{q ; d_{R T}(p, q)<\varepsilon+1\right\}$. Hence for any point $\mathrm{p}$, the $R T_{1}$ neighbourhood of $\mathrm{p}$ is defined as $N_{d_{R T}, 1}(p)=$ $\left\{q ; d_{R T}(p, q)<2\right\}$ and the $R T_{2}$ neighbourhood of $\mathrm{p}$ is defined as $N_{d_{R T}, 2}(p)=\left\{q ; d_{R T}(p, q)<3\right\}$ $N_{d_{R T: 1}}(p)$ is also denoted by $R_{3}(p)$. The $R T_{1}$ neighbours of $\mathrm{p}$ are $N_{d_{R T}, 1}(p)-\{p\}$ and the $R T_{2}$ neighbours of p are $N_{d_{R T}, 2}(p)-\{p\}$.

It is seen that the $V_{1}, 1-H, L T_{1}$ and $R T_{1}$ neighbourhood has 3 points and the $V_{2}, 2-H, L T_{2}$ and $R T_{2}$ neighbourhood has 5 points.

\section{SOME EXISTING FILTERING TECHNIQUES}

In this section, we provide the definitions of some existing filters. The image processing function in a spatial domain can be expressed as $\mathrm{g}(\mathrm{p})=\boldsymbol{\gamma}(\mathrm{f}(\mathrm{p}))$, where $\boldsymbol{\gamma}$ is the transformation function, $f(p)$ is the pixel value (gray level value) of the point $\mathrm{p}(\mathrm{x}, \mathrm{y})$ of the input image and $\mathrm{g}(\mathrm{p})$ is the pixel value of the corresponding point of the processed image.

\subsection{Median Filter [1]}

The median filter plays an important role in image processing and vision. It is useful for reducing salt and pepper noise in an image. In median filter, the pixel value of a point $p$ is replaced by the median of pixel values of 8 -neighbourhood of a point ' $\mathrm{p}$ '. The operation of this filter can be expressed as:

$g(p)=\operatorname{median}\left\{f(p)\right.$, where $\left.p \in N_{d_{8}, 1}(p)\right\}$.

\subsection{Center Weighted Median Filter [6]}

Center weighted median filter is a filter that gives more weight to the center pixel. This weight corresponds to the size of the kernel. If the kernel is $3 \times 3$, then the total number of pixels that will be converting to array for performing sorting will be 11. This filter is defined as $g(p)=\operatorname{median}\left\{\begin{array}{c}2 \diamond f\left(p_{c}\right), \\ f(p) \text { where } p \in N_{d_{8}, 1}(p)\end{array}\right\}$,

where $\checkmark$ represents replication operator and $f\left(p_{c}\right)$ is gray level value of the center pixel.

\subsection{Center Weighted Hybrid Max}

\section{(CW Hybrid Max) Filter [6]}

In Max filter [8] each output pixel value can be calculated by selecting maximum gray level value of $N_{8}(p)$. The Center Weighted Hybrid Max filter is defined as

$g(p)=$

$\max \left\{\begin{array}{c}\text { median }\left\{2 \diamond f\left(p_{c}\right), f(p) \text { where } p \in N_{d_{L T, 1}}(p)\right\}, \\ \text { median }\left\{2 \diamond f\left(p_{c}\right), f(p) \text { where } p \in N_{d_{R T, 1}}(p)\right\}, \\ f(p)\end{array}\right\}$

where $\theta$ represents replication operator and $f\left(p_{c}\right)$ is gray level value of the center pixel.

\section{PROPOSED FILTERING TECHNIQUES}

\section{1 $V_{\varepsilon}$ Median Filter}

In $V_{\varepsilon}$ Median filter, the pixel value of a point $\mathrm{p}$ is replaced by the median of the pixel values of $V_{\varepsilon}$ neighbourhood of $\mathrm{p}$. The operation of this filter can be expressed as $g(p)=\operatorname{median}\left\{f(p)\right.$, where $\left.p \in N_{d_{V}, \varepsilon}(p)\right\}$

\section{$4.2 \varepsilon-H$ Median Filter}

In $\varepsilon-H$ Median filter, the pixel value of a point $\mathrm{p}$ is replaced by the median of the pixel values of $\varepsilon-\mathrm{H}$ neighbourhood of $\mathrm{p}$. The operation of this filter can be expressed as

$g(p)=$ median $\left\{f(p)\right.$, where $\left.p \in N_{d_{H}, \varepsilon}(p)\right\}$.

\subsection{LTE Median Filter}

In $L T_{\varepsilon} \quad$ Median filter, the pixel value of a point $\mathrm{p}$ is replaced by the median of the pixel values of $L T_{\varepsilon}$ neighbourhood of $\mathrm{p}$. The operation of this filter can be expressed as

$g(p)=\operatorname{median}\left\{f(p)\right.$, where $\left.p \in N_{d_{L T, \varepsilon}}(p)\right\}$.

\subsection{RTE Median Filter}

In $R T_{\varepsilon}$ Median filter, the pixel value of a point $\mathrm{p}$ is replaced by the median of the pixel values of $R T_{\varepsilon}$ neighbourhood of $\mathrm{p}$. The operation of this filter can be expressed as $g(p)=$ median $\left\{f(p)\right.$, where $\left.p \in N_{d_{R T}, \varepsilon}(p)\right\}$.

\subsection{Center Weighted VE $(C W V \varepsilon)$}

\section{Median Filter}

Center Weighted $V_{\varepsilon}$ Median Filter is a filter that gives more weight to the center pixel of the $V_{\varepsilon}$ neighbourhood of $\mathrm{p}$. In this filter, the pixel value of a point $\mathrm{p}$ is replaced by the median of pixel values of 2 times $f\left(p_{c}\right)$ and the $V_{\varepsilon}$ neighbourhood of $\mathrm{p}$. This filter is defined 
as

$$
g(p)=\operatorname{median}\left\{\begin{array}{c}
2 \diamond f\left(p_{c}\right), \\
f(p) \text { where } p \in N_{d_{V, \varepsilon}}(p)
\end{array}\right\}
$$

where represents replication operator and $f\left(p_{c}\right)$ is gray level value of the center pixel.

\subsection{Center Weighted $\varepsilon-\mathrm{H}(\mathrm{CW} \varepsilon-\mathrm{H})$ Median Filter}

Center Weighted $\varepsilon-\mathrm{H}$ Median Filter is a filter that gives more weight to the center pixel of the $\varepsilon-\mathrm{H}$ neighbourhood of $\mathrm{p}$. In this filter, the pixel value of a point $p$ is replaced by the median of pixel values of 2 times $f\left(p_{c}\right)$ and the $\varepsilon-\mathrm{H}$ neighbourhood of p. This filter is defined as

$g(p)=$ median $\left\{\begin{array}{c}2 \diamond f\left(p_{c}\right), \\ f(p) \text { where } p \in N_{d_{H}, \varepsilon}(p)\end{array}\right\}$,

where $\theta$ represents replication operator and $f\left(p_{c}\right)$ is gray level value of the center pixel. Here we have used the notation $\varepsilon-\mathrm{H}$ instead of $H_{\varepsilon}$ to differentiate the Hybrid filters introduced by Gnanambal Ilango and R. Marudhachalam [5].

\subsection{Center Weighted $\mathrm{LT}_{\varepsilon}\left(\mathrm{CWLT}_{\varepsilon}\right)$ Median Filter}

Center Weighted $L T_{\mathcal{\varepsilon}}$ Median Filter is a filter that gives more weight to the center pixel of the $L T_{\varepsilon}$ neighbourhood of $\mathrm{p}$. In this filter, the pixel value of a point $\mathrm{p}$ is replaced by the median of pixel values of 2 times $f\left(p_{c}\right)$ and the $L T_{\varepsilon}$ neighbourhood of p. This filter is defined as

$g(p)=\operatorname{median}\left\{\begin{array}{c}2 \diamond f\left(p_{c}\right), \\ f(p) \text { where } p \in N_{d_{L T, \varepsilon}}(p)\end{array}\right\}$,

where $\theta$ represents replication operator and $f\left(p_{c}\right)$ is gray level value of the center pixel.

\subsection{Center Weighted $\mathrm{RT}_{\varepsilon}\left(\mathrm{CWRT}_{\varepsilon}\right)$ Median Filter}

Center Weighted $R T_{\varepsilon}$ Median Filter is a filter that gives more weight to the center pixel of the $R T_{\varepsilon}$ neighbourhood of $\mathrm{p}$. In this filter, the pixel value of a point $\mathrm{p}$ is replaced by the median of pixel values of 2 times $f\left(p_{c}\right)$ and the $R T_{\varepsilon}$ neighbourhood of $\mathrm{p}$. This filter is defined as

$$
g(p)=\text { median }\left\{\begin{array}{c}
2 \diamond f\left(p_{c}\right), \\
f(p) \text { where } p \in N_{d_{R T}, \varepsilon}(p)
\end{array}\right\},
$$

where $\theta$ represents replication operator and $f\left(p_{c}\right)$ is gray level value of the center pixel.

\section{EXPERIMENTAL WORK}

In this work, CT image of liver infected by bacteria was taken from www.google.com - RadiologyInfo.org - Images/videos CT images. For that image speckle noise of different variances were added. For de-noising the noisy images, the proposed filters based on $\varepsilon$ - neighbourhood were applied. The filtering techniques are implemented using

MATLAB 7.0. The performance of all the eight new filtering techniques are analyzed and discussed. The enhancement of CT images were evaluated by using statistical tools Root Mean Square Error (RMSE) and Peak Signal to Noise Ratio (PSNR).

$$
\begin{aligned}
R M S E= & \sqrt{\frac{\sum[f(i, j)-g(i, j)]^{2}}{m n}} \\
& P S N R=20 \log _{10}\left(\frac{255}{R M S E}\right)
\end{aligned}
$$

Here $f(i, j)$ is pixel value of original CT image, $g(i, j)$ is pixel value of enhanced CT image and $m$ and $n$ are the total number of pixels in the horizontal and vertical dimensions of the image. If the value of RMSE is low and the value of the PSNR is high, then the enhancement approach is better. The CT image of liver with speckle noise and filtered image obtained by the proposed filters are compared and analyzed.

\section{RESULT ANALYSIS AND DISCUSSION}

The experimental results shows that the $\varepsilon$ - neighbourhood median filters for $\varepsilon=1$ are better than the $\varepsilon$ - neighbourhood median filters for $\varepsilon=2$. Also the results indicate that the center weighted $\varepsilon$ - neighbourhood median filters perform better than the corresponding median filters. In particular, the center weighted $L T_{1}$ median filter is better than the other proposed median filters as well as it is better than the center weighted hybrid max filter introduced by R. Marudhachalam and Gnanambal Ilango [6].

Table-1 shows the RMSE and PSNR values of different proposed filters for $\mathrm{CT}$ image of liver corrupted by speckle noise with variances $0.01,0.02,0.03,0.04$ and 0.05 .

Fig. 1 shows the original image. Fig. 2 shows the noisy image. Fig. 3 shows the images obtained by applying the proposed filtering techniques for the CT image of liver with speckle noise of variance 0.01

Chart-1 shows the analysis of RMSE and PSNR values of CT liver image corrupted by speckle noise of variance 0.01 . 
Table-1 : RMSE and PSNR values of different proposed filters for CT image of Liver corrupted by speckle noise with variances $0.01,0.02,0.03,0.04$ and 0.05 .

\begin{tabular}{|c|c|c|c|c|c|c|c|c|c|c|}
\hline \multirow{3}{*}{ Filters } & \multicolumn{10}{|c|}{ Variance } \\
\hline & \multicolumn{2}{|c|}{0.01} & \multicolumn{2}{|c|}{0.02} & \multicolumn{2}{|c|}{0.03} & \multicolumn{2}{|c|}{0.04} & \multicolumn{2}{|c|}{0.05} \\
\hline & RMSE & PSNR & RMSE & PSNR & RMSE & PSNR & RMSE & PSNR & RMSE & PSNR \\
\hline$V_{1}$ Median & 5.1683 & 33.8642 & 5.6031 & 33.1627 & 5.8150 & 32.8402 & 5.9633 & 32.6214 & 6.0830 & 32.4489 \\
\hline$\nu_{2}$ Median & 6.0796 & 32.4537 & 6.5125 & 31.8562 & 6.7615 & 31.5304 & 6.9027 & 31.3508 & 7.0155 & 31.2101 \\
\hline $1-H$ Median & 5.3576 & 33.5518 & 5.7445 & 32.9461 & 5.9537 & 32.6355 & 6.0526 & 32.4923 & 6.1908 & 32.2963 \\
\hline 2-H Median & 6.3525 & 32.0723 & 6.7283 & 31.5731 & 6.8971 & 31.3579 & 7.0595 & 31.1558 & 7.1538 & 31.0405 \\
\hline$L T_{1}$ Median & 5.4951 & 33.3318 & 5.8651 & 32.7657 & 6.0693 & 32.4685 & 6.1885 & 32.2996 & 6.2912 & 32.1565 \\
\hline$L T_{2}$ Median & 6.4441 & 31.9479 & 6.8379 & 31.4327 & 7.0510 & 31.1662 & 7.1594 & 31.0337 & 7.2455 & 30.9298 \\
\hline$R T_{1}$ Median & 6.4893 & 31.8873 & 6.8560 & 31.4098 & 7.0581 & 31.1574 & 7.1991 & 30.9856 & 7.2605 & 30.9119 \\
\hline$R T_{2}$ Median & 6.4893 & 31.8873 & 6.8560 & 31.4098 & 7.0581 & 31.1574 & 7.1991 & 30.9856 & 7.2605 & 30.9119 \\
\hline$C W V$, Medion & 0.2534 & 60.0556 & 0.2473 & 60.2658 & 0.2466 & 60.2912 & 0.2480 & 60.2434 & 0.2531 & 60.0663 \\
\hline $\mathrm{CWV}_{2}$ Median & 3.7537 & 36.6421 & 4.0360 & 36.0122 & 4.1995 & 35.6672 & 4.3176 & 35.4264 & 4.4231 & 35.2167 \\
\hline$C W 1-H$ Median & 0.2504 & 60.1588 & 0.2539 & 60.0382 & 0.2468 & 60.2842 & 0.2519 & 60.1082 & 0.2407 & 60.5016 \\
\hline$C W 2$ - $H$ Median & 3.8906 & 36.3309 & 4.2309 & 35.6026 & 4.3710 & 35.3197 & 4.5124 & 35.0430 & 4.5567 & 34.9582 \\
\hline$C W L T_{1}$ Median & 0.2480 & 60.2420 & 0.2513 & 60.1273 & 0.2473 & 60.2686 & 0.2470 & 60.2785 & 0.2533 & 60.0596 \\
\hline$C W L T_{2}$ Median & 4.0361 & 36.0120 & 4.3457 & 35.3700 & 4.4726 & 35.1200 & 4.5990 & 34.8781 & 4.6367 & 34.8070 \\
\hline CWRT, Median & 0.2491 & 60.2057 & 0.2536 & 60.0489 & 0.2549 & 60.0036 & 0.2473 & 60.2686 & 0.2526 & 60.0825 \\
\hline$C W R T_{2}$ Median & 4.0672 & 35.9454 & 4.3482 & 35.3651 & 4.5357 & 34.9983 & 4.6219 & 34.8348 & 4.7033 & 34.6832 \\
\hline CW Hybrid Max & 0.4911 & 54.3075 & 0.5075 & 54.0222 & 0.5025 & 54.1096 & 0.5152 & 53.8925 & 0.5163 & 53.9140 \\
\hline
\end{tabular}




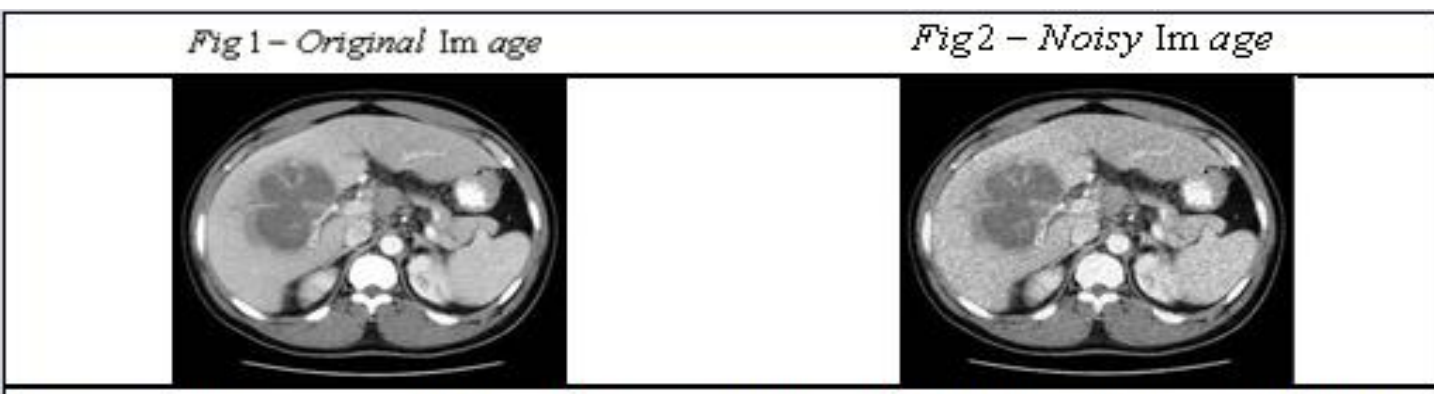

Fig 3 - Images obtained by the proposed filtering techniques for the CT image of liver with speckle noise of variance 0.01
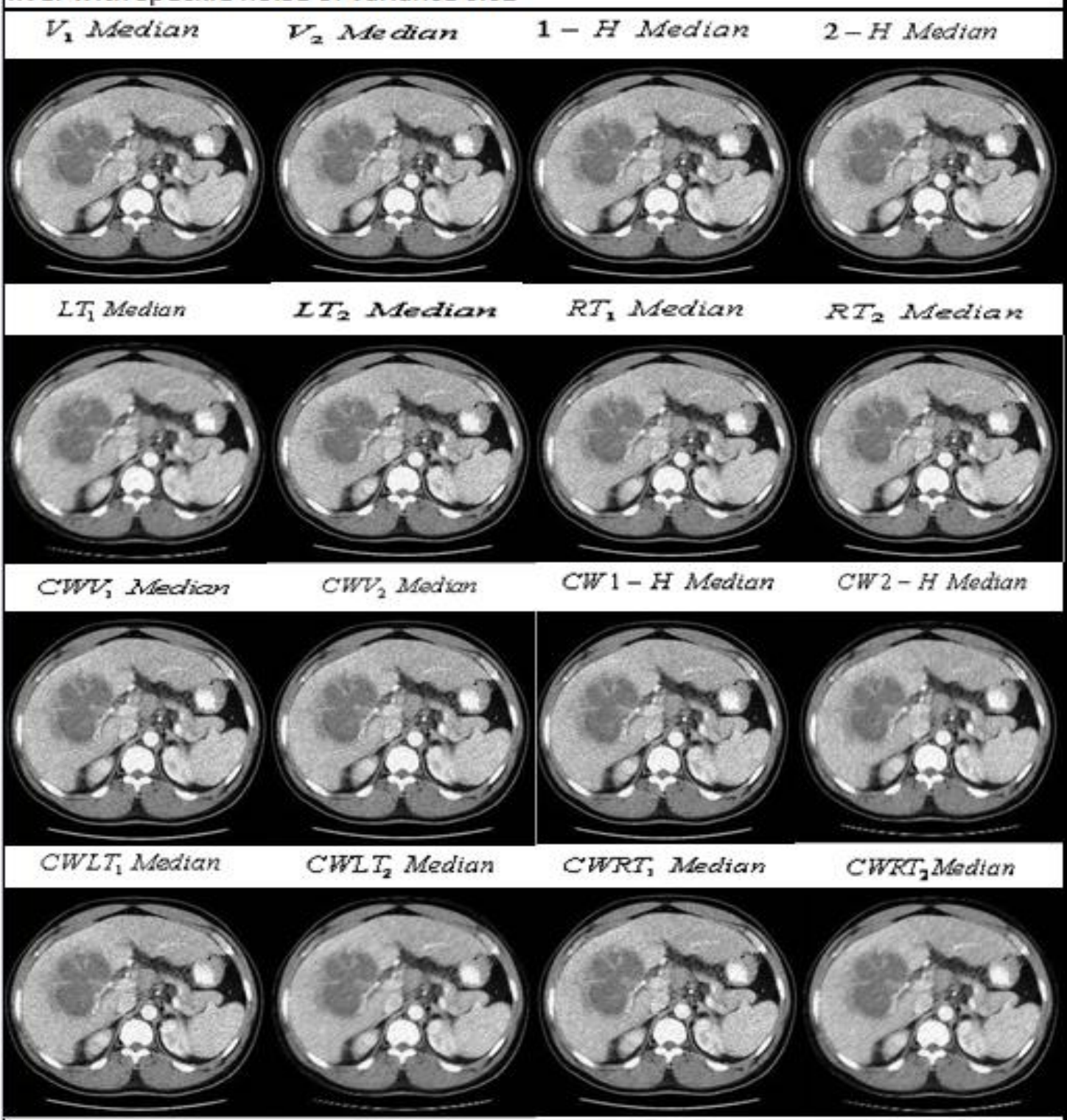

CW Hybrid Max

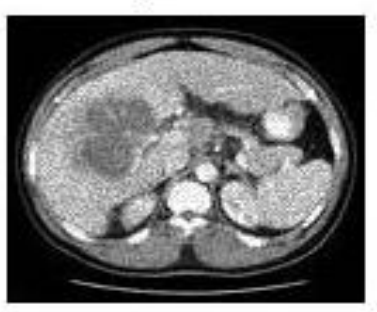




\section{Chart1: Analysis of RMSE and PSNR values of CT liver image corrupted by speckle noise of variance 0.01}
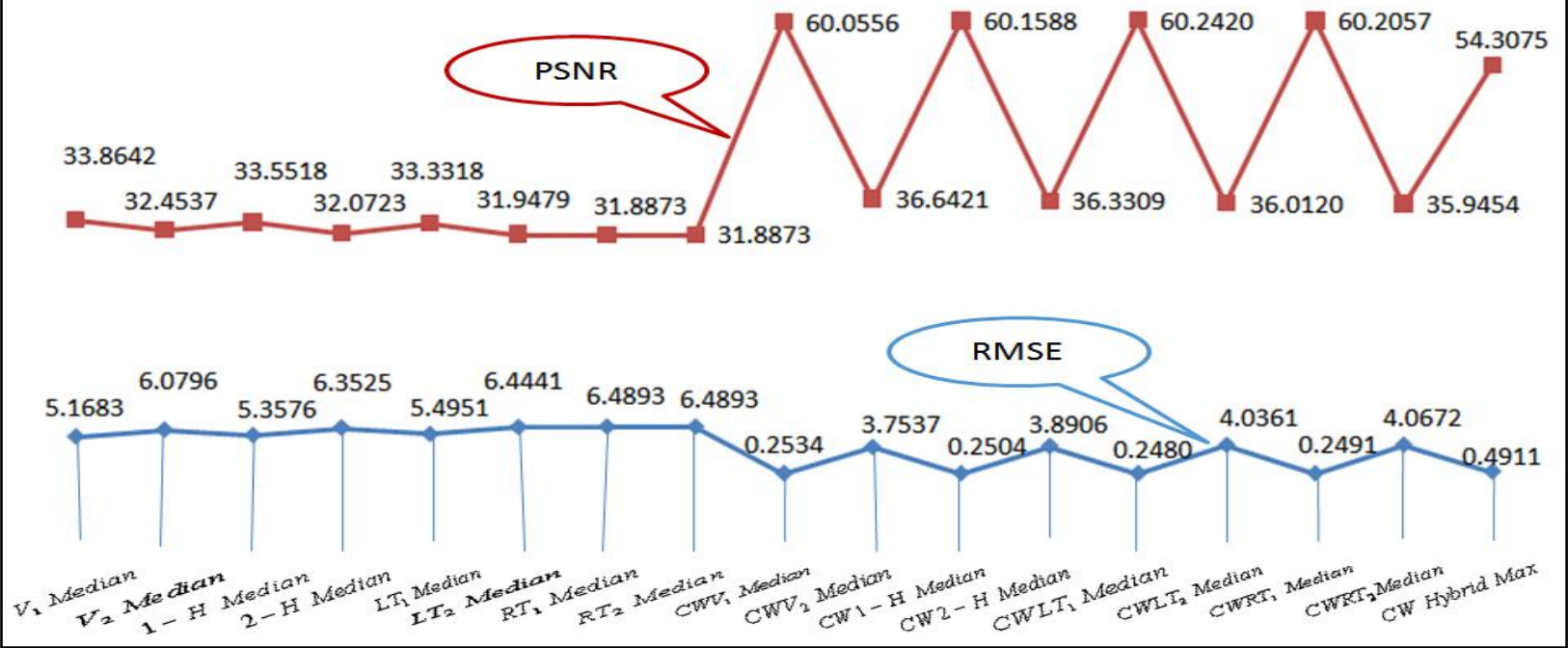

\section{CONCLUSION}

In this work, we have introduced eight new filtering techniques for the removal of speckle noise from CT images, by metric topological neighbourhood approach. To demonstrate the performance of the proposed techniques, the experiments have been conducted on CT image of liver. The performance of speckle noise removal by proposed filtering techniques is measured using quantitative performance measures such as RMSE and PSNR. The experimental results indicate that the center weighted $L T_{1}$ median filter performs significantly better than the other proposed filters as well as it is better than the Center Weighted Hybrid Max filter introduced by R. Marudhachalam and Gnanambal Ilango [6]. This result has been verified for some more CT and MRI images.

\section{REFERENCES}

[1] J.W.Turkey, 1974. Nonlinear (nonsuperposable) methods for smoothing data, Proc. Congr. Rec. EASCOM'74, 673-681.

[2] S. Sudha, G. R. Suresh and R. Sukanesh, 2009. Speckle noise reduction in Ultrasound Images by Wavelet thresholding based on Weighted variance, International Journal of Comp. Theory and Engg, No.1, 7-12.

[3] Mamta Juneja and Rajni Mohana, 2009. An improved Adaptive Median Filtering Method for Impulse Noise Detection, International Journal of Recent Trends in Engineering, No.1, 274-278.
[4] K. Thangavel, R. Manavalan, and I. Laurence Aroquiaraj, 2009. Removal of Speckle noise from Ultrasound Medical Image based on Special Filters: Comparative Study, International Conference on Graphics, Vision and Image Processing, 25-32.

[5] Gnanambal Ilango and R. Marudhachalam, 2011. New hybrid filtering techniques for removal of speckle noise from ultrasound medical images, Scientia Magna, vol. 7 , No. 1, 38-53.

[6] R. Marudhachalam and Gnanambal Ilango, 2011. Center Weighted Hybrid Filtering Techniques for denoising of medical images, World Congress on Engineering and Technology, 542-545.

[7] James R. Munkres, 2007. Topology, Prentice-Hall of India.

[8] R. Gonzalez and R. Woods, 1992. Digital Image Processing, Adison-Wesley, New York.

[9] R. Klette and A. Rosenfeld, 2004. Digital Geometry, Kaufmann, San Francisco. 